\title{
TITLE:
}

\section{The Levi Problem and the Structure Theorem for Non-Negatively Curved Complete Kahler Manifolds}

\author{
AUTHOR(S): \\ Takayama, Shigeharu
}

\section{CITATION:}

Takayama, Shigeharu. The Levi Problem and the Structure Theorem for Non-Negatively Curved Complete Kahler Manifolds. 数理解析研究所講究録 1998, 1058: 105-113

\section{ISSUE DATE:}

1998-08

URL:

http://hdl.handle.net/2433/62328

RIGHT: 
The Levi Problem and the Structure Theorem

for Non-Negatively Curved Complete Kähler Manifolds

Shigeharu TAKAYAMA： 高山 茂晴

Naruto Univ. Edu. / Osaka Univ.

\section{$\S 1$. Introduction and Statement of Result.}

Recent development of complex geometry enable us to get global sections of an adjoint bundle on a projective manifold, under a reasonable numerical condition [AS] [D2] [Tj]. The theory which is mainly developed by Demailly and Siu, is very concrete and constructive. Hence their method can be applied in various contexts [Ty2] [Ty4]. Here we apply their method to construct holomorphic functions on certain pseudoconvex manifolds.

\section{Levi problem on a complex manifold.}

Let us consider the following function theoritic properties of a complex manifold $X$ :

(i) $X$ is holomorphically convex;

(ii) there exists a proper holomorphic map $X \longrightarrow \mathbf{C}^{N}$;

(iii) $X$ is weakly 1-complete, i.e., there exists a smooth function $\Phi$ : $X \longrightarrow \mathbf{R}$ which is plurisubharmonic and exhaustive.

(iv) $X$ is pseudoconvex, i.e., there exists a continuous plurisubharmonic exhaustion function.

It is well known that implications (i) $\Leftrightarrow$ (ii) $\Rightarrow$ (iii) $\Rightarrow$ (iv) hold. Then the Levi problem asks whether the implication (iv) $\Rightarrow$ (i) (or (iii) $\Rightarrow$ (i)) holds or not.

If $X$ is a domain in $\mathbf{C}^{n}$, the answer is affirmative. However, as we will see below, there exists a quotient complex manifold of $\mathbf{C}^{n}$ such that it is weakly 1-complete but it is not holomorphically convex. Therefore we need some condition to get affirmative results in general. Here we impose a condition on the canonical bundle. The motive of this work in the following 
Theorem 1.1 Ohsawa [O]. Let $X$ be a 2-dimensional complex manifold with a negative canonical bundle $K_{X}$. Then $X$ is holomorphically convex if and only if it is weakly 1-complete.

Here we generalize Ohsawa's theorem for higher dimensional cases as follows:

Main Theorem 1.2. Let $X$ be a complex manifold with a negative canonical bundle $K_{X}$. Then $X$ is holomorphically convex if and only if it is pseudoconvex.

The proof depends on effective construction of singular Hermitian metric and related vanishing theorem.

Structure theorem for non-negatively curved complete Kähler manifold. There are relations of metric properties of manifolds and the pseudoconvexity. We apply our Main Theorem to study such relations. Let us recall Riemannian case [CG].

Cheeger-Gromoll: Let $(M, g)$ a complete Riemannian manifold with non-negative sectional curvature. Then there exists a totally geodesic compact submanifold $S$ such that $M \approx N_{S / M}$, i.e., $M$ is diffeomorphic to the total space of the normal bundle of $S$ in $M$.

Their key result: basic construction [CG, §1] claim that, by using Busemann's function, a complete Riemannian manifold with non-negative sectional curvature has a continuous geodesically convex exhaustion function. Inspired by their works, Greene-Wu [GW] studied non-negatively curved Kähler manifolds.

Greene-Wu: Let $(X, g)$ a complete Kähler manifold with positive (resp. non-negative) sectional curvature. Then there exists a continuous strictly plurisubharmonic (resp. plurisubharmonic) exhaustion function. Their main conclusion is as follows: Let $(X, g)$ as above with positive sectional curvature, then $X$ is Stein and diffeomorphic to $\mathbf{C}^{n}$. They also asked a kind of the Levi problem: 
Conjecture 1.3 (Greene-Wu [GW]). Every complete Kähler manifold with non-negative sectional curvature and positive Ricci curvature is holomorphically convex.

Since a Kähler manifold $(X, g)$ as in Conjecture 1.3 is pseudoconvex and the canonical bundle is negative, we can solve Conjecture 1.3 affirmatively by our Main Theorem. It means that there exists a proper holomorphic map: the Remmert reduction $R: X \longrightarrow Y$ to a Stein space. Moreover since $X$ has semi-positive holomorphic tangent bundle, such a holomorphic reduction has very neat structure; for example, as in the structure theorem of Demailly-Peternell-Schneider [DPS]. Actually we can show that the Remmert reduction is a smooth holomorphic map over a Stein manifold, and that every fibre has a Kähler metric with nonnegative sectional curvature with positive Ricci curvature. Then by the uniformization theorem of Mok $[\mathrm{M}]$ and the rigidity theorem of Bott $[\mathrm{B}]$, we can show

Theorem 1.4. Every complete Kähler manifold with non-negative sectional curvature and positive Ricci curvature, has a structure of holomorphic fiber bundle over a Stein mainfold whose typical fibre is biholomorphic to some compact Hermitian symmetric manifold.

The following example will explain on the positivity condition of Ricci curvature (or that of anti-canonical bundle), and differences from the Riemannian case. Let us consider a quotient complex Lie group $X_{a}:=$ $\mathbf{C}^{2} / \Gamma_{a}$ of $\mathbf{C}^{2}$ by a rank 3 discrete subgroup

$$
\Gamma_{a}=\left(\begin{array}{l}
1 \\
0
\end{array}\right) \mathbf{Z}+\left(\begin{array}{l}
0 \\
1
\end{array}\right) \mathbf{Z}+\left(\begin{array}{c}
\sqrt{-1} \\
a
\end{array}\right) \mathbf{Z} \text { with } a \in \mathbf{R} .
$$

Then every $X_{a}$ is a non-compact, weakly 1-complete Kähler manifold with a trivial (holomorphic) tangent bundle $T_{X_{a}} \cong \mathcal{O}_{X_{a}} \oplus \mathcal{O}_{X_{a}}$ (cf. [K1]). As real Lie groups, they have very simple strucure $X_{a} \cong T_{\mathbf{R}}^{3} \times \mathbf{R}$. On the other hand, their complex structures are very subtle $[\mathrm{K} 2]$. By an elementary argument we see the following four conditions are equivalent 
to each other:

(i) $X_{a}$ has a non-constant holomorphic function;

(ii) $X_{a}$ is a product of an elliptic curve and $\mathbf{C}^{*}$;

(iii) there exists a compact complex curve in $X_{a}$;

(iv) $a$ is rational.

It may happen $H^{1}\left(X_{a}, \mathcal{O}_{X_{a}}\right)$ is non-Hausdorff, if $a$ belongs to a certain class of transcendental numbers. Although our approach can be applied to establish Lefschetz type theorems on certain kind of such non-compact quotients $\mathbf{C}^{n} / \Gamma$, so-called quasi-abelian varieties [Ty3].

\section{§2. Vanishing Theorem.}

Let us recall basic tools and notions [D1] [D2]. Let $X$ a complex manifold, $(L, h)$ a Hermitian holomorphic line bundle on $X$ with positive curvature $\operatorname{curv} h:=\sqrt{-1} \partial \bar{\partial} \log h>0$. Let $\beta$ a positive rational number, $s=\left\{s_{j}\right\}_{j \in J}$ a finite number of multivalued holomorphic sections of $L^{\otimes \beta}$ on $X$. The multiplier ideal sheaf $\mathcal{I}(s)$ of $s$ is defined as follows: for every open set $U$,

$$
\mathcal{I}(s)(U):=\left\{f \in H^{0}(U, \mathcal{O}) ; \int_{U}|f|^{2}\left(|s|^{2}\right)^{-1} d v<+\infty\right\} .
$$

We see $\mathcal{I}(s)$ is a coherent ideal sheaf of $\mathcal{O}_{X}$. We denote $V \mathcal{I}(s):=\mathcal{O}_{X} / \mathcal{I}(s)$ the closed complex subspace of $X$ definied by $\mathcal{I}(s)$. For every integer $m>\beta$, we define a singular Hermitian metric of $L^{\otimes m}$ by

$$
H_{m}:=\frac{h^{m}}{|s|^{2}}=h^{m-\beta} \frac{h^{\beta}}{|s|^{2}} .
$$

We see, by the local computation, the curvature current satisfies

$$
\operatorname{curv} H_{m} \geq(m-\beta) \operatorname{curv} h>0 .
$$

We also define $\mathcal{I}\left(H_{m}\right):=\mathcal{I}(s)$ the multiplier ideal sheaf of $H_{m}$. Now we recall

Demailly's Nadel vanishing theorem 2.1. Assume that $X$ is pseudoconvex. Then $H^{1}\left(X, K_{X} \otimes L^{\otimes m} \otimes \mathcal{I}\left(H_{m}\right)\right)=0$. As a consequence, the restriction map

$$
H^{0}\left(X, K_{X} \otimes L^{\otimes m}\right) \longrightarrow H^{0}\left(V \mathcal{I}(s), K_{X} \otimes L^{\otimes m} \otimes \mathcal{O}_{X} / \mathcal{I}(s)\right)
$$


is surjective.

If we have sections on $V \mathcal{I}(s)$, then we have global sections of $K_{X} \otimes L^{\otimes m}$. This is rather abstruct existence theorem. We have to controle the tensor power $m$ and the locus $V \mathcal{I}(s)$. A zero-dimensional subspace is a candidate of a good locus, but then it is hard to controle the tensor power $m$. In the next section, we do effective construction of singular Hermitian metrics for another candidate of a locus which has a nice property.

\section{§3. Effective Vanishing Theorem.}

We let $X$ a non-compact pseudoconvex manifold with a continuous plurisubharmonic exhaustion function $\Phi: X \longrightarrow \mathbf{R}$, and let $(L, h)$ a positive line bundle on it. We consider the following equivalent relation on $X$ : Let $x, y \in X$. Then " $x \sim y$ " iff $x$ and $y$ are joined by a finite number of irreducible compact complex subspaces of $X$. We take the quotient $R: X \longrightarrow R X:=X / \sim$, as sets, which we call the formal Remmert reduction of $X$.

\section{Remark 3.1.}

(1) If $X$ is holomorphically convex, then $R: X \longrightarrow R X$ is the Remmert reduction.

(2) the plurisubharmonic function $\Phi$ is constant along each fibre of $R$.

(3) $R X$ is not a point.

A fibre is a candidate of a good locus. We would like to separate two distinct fibres of $R$ by holomorphic functions.

Let us take two distinct points $x_{i}^{\prime} \in R X(i=1,2)$, and set $V_{i}:=$ $R^{-1}\left(x_{i}^{\prime}\right)$. We note that every $V_{i}$ is a relatively compact set of $X$ and that $V_{1} \cap V_{2}=\emptyset$. Take a sublevel set $X_{c}:=\{x \in X ; \Phi(x)<c\}$ of $(X, \Phi)$ containing $V_{1}$ and $V_{2}$. Our main technical result is as follows.

Theorem 3.2. Let $\beta$ be a rational number with $0<\beta<1$. Then there exist a finite number of multivalued holomorphic sections $s=\left\{s_{j}\right\}_{j \in J}$ of $L^{\otimes \beta}$ on $X$ with the following three properties: 
(1) $V \mathcal{I}(s) \cap V_{i} \neq \emptyset(i=1,2)$;

(2) $V \mathcal{I}(s)$ has only one irreducible component $Z$ which intersects $V_{1}$;

(3) The irreducible component $Z$ in (2) is compact.

We can decompose $V \mathcal{I}(s)=Z \amalg V$ with $Z$ is compact and $Z \subset V_{1}$; $V \cap V_{2} \neq \emptyset$ but $V \cap V_{1}=\emptyset$. Then applying Demailly's Nadel vanishing theorem 2.1, we have

Corollary 3.3. The restriction map

$$
H^{0}\left(X_{c}, K_{X} \otimes L\right) \longrightarrow H^{0}\left(Z, K_{X} \otimes L \otimes \mathcal{O}_{Z}\right) \oplus H^{0}\left(V, K_{X} \otimes L \otimes \mathcal{O}_{V}\right)
$$

is surjective.

We do not know existence of sections. However in a special case, we do have. For example the formal Remmert reduction is bijective, another example is negative canonical bundle case as follows.

\section{$\S 4$. Levi Problem.}

Let us go back to the original problem. We let $(X, \Phi)$ be a noncompact pseudoconvex manifold with negative canonical bundle $K_{X}$. We take $L=K_{X}^{\otimes(-1)}$ in $\S 3$. We use the same notation as in $\S 3$. Then by Corollary 3.3 we have a surjection

$$
H^{0}\left(X_{c}, \mathcal{O}\right) \longrightarrow H^{0}\left(Z, \mathcal{O}_{Z}\right) \oplus H^{0}\left(V, \mathcal{O}_{V}\right)
$$

We extend a holomorphic function $(1,0) \in H^{0}\left(Z, \mathcal{O}_{Z}\right) \oplus H^{0}\left(V, \mathcal{O}_{V}\right)$ on $X_{c}$. Since every holomorphic function is constant along the fibre of $R$ : $X \longrightarrow R X$, there exists a holomorphic function $f \in H^{0}\left(X_{c}, \mathcal{O}\right)$ such that $\left.f\right|_{V_{1}} \equiv 1$ and $\left.f\right|_{V_{2}} \equiv 0$. We can separate every pair of two distinct fibres of $R$ by a holomorphic function on $X_{c}$. Then after some arguments we see every $X_{c}$ is holomorphically convex, and then by Narasimhan's approximation theorem $[\mathrm{Nr}], X$ is holomorphically convex.

\section{$\S 5$. Example of Construction.}

We consider 2-dimensional case in $\S 3$. We set $n=2$. Let us take a rational number $\varepsilon_{0}$ with $0<\varepsilon_{0}<\beta$, and positive integers $p$ and $q$ 
such that $n p / q<\varepsilon_{0}$. By the so-called line bundle convexity property of the sublevel set $X_{c}$ with respect to the positive line bundle $L$, we see $\operatorname{dim} H^{0}\left(X_{c}, L^{\otimes m}\right)=+\infty$ for every large $m$. We take a point $x_{i} \in V_{i}$ $(i=1,2)$. Then we can take a non-zero section

$$
\sigma \in H^{0}\left(X_{c}, L^{\otimes p m} \otimes \mathcal{M}_{x_{1}}^{m q} \mathcal{M}_{x_{2}}^{m q}\right)
$$

here $\mathcal{M}_{x}$ is the maximal ideal sheaf of $x$ in $X$. We consider (log-canonical thresholds)

$$
\begin{aligned}
\alpha_{i} & :=\sup \left\{t \geq 0 ; V \mathcal{I}\left(\sigma^{t n /(m q)}\right) \cap V_{i}=\emptyset\right\} \\
\alpha & :=\max \left\{\alpha_{1}, \alpha_{2}\right\} .
\end{aligned}
$$

We see every $\alpha_{i}$ is a rational number with $0<\alpha_{i} \leq 1$. The section $\sigma_{1}:=\sigma^{\alpha n /(m q)}$ may be desired one. If it is not, we continue the above procedure on the locus $V \mathcal{I}\left(\sigma_{1}\right)$. Let $V \mathcal{I}\left(\sigma_{1}\right)=\cup Y_{i}$ be the irreducible decomposition. For example the following cases may happen:

(1) $\alpha_{1}=\alpha_{2}, x_{1}, x_{2} \in Y_{1}$ but $\left(V_{1} \cup V_{2}\right) \cap Y_{i}=\emptyset$ for any $i \neq 1$;

(2) $\alpha_{1}>\alpha_{2}, x_{1} \in Y_{1} \not \subset V_{1}$ but $V_{1} \cap Y_{i}=\emptyset$ for any $i \neq 1$.

We should note that these $Y_{1}$ are non-compact.

We consider the first case. After replacing $X_{c}$ by a smaller sublevel set of $(X, \Phi)$, we can take a multivalued holomorphic section $\tau$ of $L^{\otimes \beta-\varepsilon_{0}}$ on $X_{c}$ such that the restriction $\left.\tau\right|_{Y_{1}}$ is not identically zero, and vanishes at $x_{1}$ and $x_{2}$ with high multiplicities in $Y_{1}$ : This follows from the line bundle convexity property of $Y_{1}$ with respect to $L$. Then, for a sufficiently small positive rational number $\delta$, we have a multivalued holomorphic section $s:=\sigma_{1}^{1-\delta} \times \tau$ of $L^{\otimes \beta^{\prime}}$ on $X_{c}$ for some $0<\beta^{\prime}<\beta$. Then we will have

$$
x_{1}, x_{2} \in V \mathcal{I}(s) \subset V \mathcal{I}\left(\sigma_{1}\right) \cap(\tau)_{0},
$$

which means $V \mathcal{I}(s)$ is zero-dimensional around $V_{1}$ and $V_{2}$. Thus we obtained a desired section $s$. Here we need the semicontinuity argument of Angehrn-Siu [AS].

References. 
[AS] Angehrn- Siu: Effective freeness and point separation for adjoint bundles, Invent. math. 122 (1995) 291-308.

[B] Bott: Homogeneous vector bundles, Ann. of Math. 66 (1957) 203-248. [CG] Cheeger- Gromoll: On the structure of complete manifolds of nonnegative curvature, Ann. Math. 96 (1972) 413-443.

[D1] Demailly: Estimations $L^{2}$ pour l'opérateur $\bar{\partial}$ d'un fibré vectoriel holomorphe semi-positif au-dessus d'une variété kählérienne complète, Ann. Sic. Éc. Norm. Sup. 15 (1982) 457-511.

[D2] Demailly: $L^{2}$ vanishing theorem for positive line bundles and adjunction theory, CIME Session, Transcendental Methods in Algebraic Geometry, Cetraro, Italy (1994) to appear.

[DPS] Demailly- Peternell- Schneider: Compact complex manifolds with numerically effective tangent bundles, J. Alg. Geom. 3 (1994) 295-345. [GW] Greene- Wu: Analysis on noncompact Kähler manifolds, Proc. Sympos. Pure Math. 30 (1977) 69-100.

[K1] Kazama: On pseudoconvexity of complex abelian Lie groups, J. Math. Soc. Japan 25 (1973) 329-333.

[K2] Kazama: $\bar{\partial}$-Cohomology of $(H, C)$-Groups, Publ. RIMS, Kyoto Univ. 20 (1984) 297-317.

[M] Mok: The uniformization theorem for compact Kähler manifolds of nonnegative holomorphic bisectional curvature, J. Diff. Geom. 27 (1988) 179-214.

[Nr] Narasimhan: The Levi Problem for Complex Spaces II, Math. Ann. 146 (1962) 195-216.

[O] Ohsawa: Weakly 1-complete manifold and Levi problem, Publ. RIMS. 17 (1981) 153-164, supplement Publ. RIMS. 17 (1981) 981-982.

[OT] Ohsawa- Takegoshi: On the Extension of L ${ }^{2}$ Holomorphic Functions, Math. Z. 195 (1987) 197-204.

[Ty1] Takayama: On Relative Base Point Freeness of Adjoint Bundle, Nagoya Math. J. 146 (1997) 185-197.

[Ty2] Takayama: Adjoint Linear Series on Weakly 1-Complete Kähler Manifolds I: Global Projective Embedding, (1996) preprint. 
[Ty3] Takayama: Adjoint Linear Series on Weakly 1-Complete Kähler Manifolds II: Lefschetz Type Theorem on Quasi-Abelian Varieties, (1996) preprint.

[Ty4] Takayama: Nonvanishing Theorems on an Algebraic Variety with Large Fundamental Group, (1997) preprint.

[Tj] Tsuji: Global generation of adjoint bundles, Nagoya Math. J. 142 (1996) 5-16.

Shigeharu TAKAYAMA

Department of Mathematics

Naruto University of Education

Takashima, Naruto-cho, Naruto-shi, 772 Japan.

e-mail address: takayama@naruto-u.ac.jp

Current address:

Department of Mathematics

Graduate School of Science

Osaka University

Toyonaka, Osaka, 560 JAPAN 